Latvian Journal of Chemistry, No 3/4, 2011, 296-307.

DOI: $10.2478 / \mathrm{v} 10161-011-0066-4$

\title{
SYNTHESIS OF SOME NEW THIAZOLIDINE DERIVATIVES AND THEIR BIOLOGICAL SIGNIFICANCE
}

\author{
R. Sharma, P. Samadhiya, S.D. Srivastava, S.K. Srivastava \\ Department of Chemistry, Dr. H.S. Gour University (A Central University), \\ Sagar, M.P. India-470003 \\ e-mail: ritusharmaic@rediffmail.com
}

An efficient route for the synthesis of new series of $\mathrm{N}-[3-(1 \mathrm{H}-1,2,3-$ benzotriazol-1-yl)-propyl]-2-(substituted phenyl)-4-oxo-5-(substituted benzylidene)-1,3-thiazolidine carboxamide, compounds $5(\mathbf{a}-\mathbf{j})$ has been elaborated. The compounds $\mathbf{5}(\mathbf{a}-\mathbf{j})$ have been synthesized and characterized by IR, ${ }^{1} \mathrm{H}$ NMR, ${ }^{13} \mathrm{C}$ NMR, FAB Mass spectra and chemical analysis. All final compounds were screened for their antimicrobial activity against some selected bacteria, fungi, antituberculosis (against $M$. tuberculosis) and antiinflammatory activity on albino rats.

Key words: synthesis, benzotriazolo-4-oxothiazolidine, antimicrobial, antitubercular activity.

\section{INTRODUCTION}

Heterocyclic compounds have been under investigation for a long time because of their important pharmacological properties. Thiazolidine possess various remarkable biological activities such as antimicrobial [1], antibacterial [2,3], antifungal [4], antipsychotic [5], antiviral [6], antitubercular [7], anticancer [8] and anti HIV [9] activity. 1,2,3-Benzotriazole derivatives have been a topic of interest of a substantial research and continue to be one of the most active areas of the heterocyclic chemistry, particularly due to their natural occurrence and pharmacological activities. The large number of benzotriazole derivatives are pharmacologically active lead compounds for drug development. Benzotriazole derivatives also occur widely in many natural products such as those from plants, fungi and marine organisms. The biological and chemical properties of the benzotriazole derivatives have attracted the attention of organic chemists, medicinal chemists, biologists and pharmacologists. Chemical and biological research has also presented a great challenge to synthesize and optimize highly efficient and economical synthetic route to novel biologically active substances.

At present there are thousands of compounds described, including simple and more complex factionalized benzotriazole derivatives. The simple benzotriazole derivatives are comprised of triazole ring fused with the benzene ring and more complex benzotriazole derivatives usually contain the additional fused rings. The benzotriazole nucleus is a pharmaceutically important and emerging heterocycle with a broad spectrum of activities including antibacterial [10-12], antifungal [13, 14], antitubercular [15] and anticancer [16] activity. We have decided to synthesize a new series of $\mathrm{N}$-[3-(1H-1,2,3-benzotriazol-1-yl)propyl]- 
2-(substituted phenyl)-4-oxo-5-(substituted benzylidene)-1,3-thiazolidine carboxamide, compounds $5(\mathbf{a}-\mathbf{j})$ ( see the Scheme).

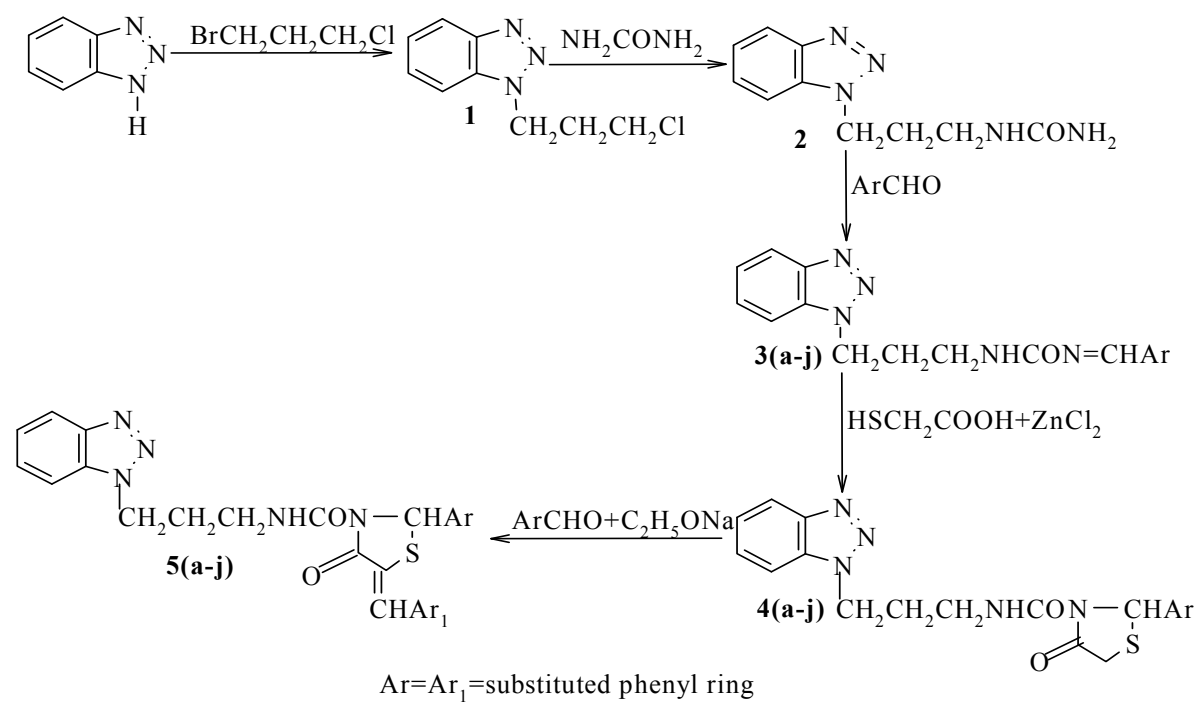

Scheme. Synthesis of compounds $\mathbf{5}(\mathbf{a}-\mathbf{j})$.

\section{EXPERIMENTAL}

Melting points were determined using open capillaries and are uncorrected. Progress of reaction was monitored by silica gel G-coated TLC plates in the $\mathrm{MeOH}: \mathrm{CHCl}_{3}(1: 9)$ system. The spots were visualized by exposing dry plate in iodine vapours. IR spectra were recorded in $\mathrm{KBr}$ discs on a Schimadzu 8201 PC, FTIR spectrophotometer $\left(v_{\max }\right.$ in $\left.\mathrm{cm}^{-1}\right)$ and ${ }^{1} \mathrm{H}$ and ${ }^{13} \mathrm{C}$ NMR spectra were measured on a Brucker DRX-300 spectrometer in $\mathrm{CDCl}_{3}$ at 300 and $75 \mathrm{MHz}$, respectively, using TMS as an internal standard. All chemical shifts were reportted on $\delta$ scale. The FAB-Mass spectra were recorded on a Jeol SX-102 mass spectrometer. Elemental analyses were performed on Carlo Erba-1108 analyzer. The analytical data for all the compounds were highly satisfactory. For column chromatographic purification of the products, Merck silica gel 60 (230400 mesh) was used. The reagent grade chemicals were purchased from the commercial sources and further purified before use.

Synthesis of 1-(3-chloropropyl)-1H-1,2,3-benzotriazole (compound 1). $1,2,3$-Benzotriazole $(0.420 \mathrm{~mol})$ and 1-bromo-3-chloropropane $(0.420 \mathrm{~mol})$ in methanol $(100 \mathrm{ml})$ were stirred on a magnetic stirrer for about 6.00 hours at room temperature. The completion of the reaction was monitored by TLC plates coated with silica gel-G. The product was filtered and purified by chromatography over a column packed with silica gel using the system $\mathrm{CHCl}_{3}: \mathrm{CH}_{3} \mathrm{OH}$ $(8: 2 \mathrm{v} / \mathrm{v})$ as eluent $(150 \mathrm{ml})$. The purified product was dried under vacuum and recrystallized from ethanol to yield the compound $\mathbf{1}$.<smiles>CCn1nnc2ccccc21</smiles> 
1-(3-Chloropropyl)-1H-1,2,3-benzotriazole (1). Yield: $62 \%$; m.p. $77-79^{\circ} \mathrm{C}$; IR spectrum $\left(\mathrm{cm}^{-1}\right)$ : $749(\mathrm{C}-\mathrm{Cl}), 1324(\mathrm{~N}-\mathrm{C}), 1463(\mathrm{C}=\mathrm{C}), 1542(\mathrm{~N}=\mathrm{N}), 1430$, 2836, $2893\left(\mathrm{CH}_{2}\right), 3021$ (CH-Ar); ${ }^{1} \mathrm{H}$ NMR spectrum (300 MHz, $\left.\mathrm{CDCl}_{3}, \mathrm{TMS}\right)$, $\delta: 2.13-2.15$ (m, 2H, H-9); 3.49 (t, 2H, $J=7.45 \mathrm{~Hz}, \mathrm{H}-10$ ); 4.17 (t, 2H, $J=7.45$ $\mathrm{Hz}, \mathrm{H}-8) ; 7.29-7.96$ (m, 4H ArH); ${ }^{13} \mathrm{C}$ NMR spectrum (75 MHz, $\mathrm{CDCl}_{3}$, TMS),

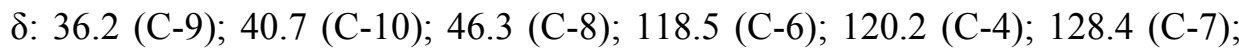
128.9 (C-5); 145.5 (C-3a); 147.9 (C-7a); FAB Mass $(\mathrm{m} / \mathrm{z})$ : $195\left[\mathrm{M}^{+}\right]$. Elemental analisys data for $\mathrm{C}_{9} \mathrm{H}_{10} \mathrm{~N}_{3} \mathrm{Cl}$ : calculated, \%: $\mathrm{C}, 55.25 ; \mathrm{H}, 5.15 ; \mathrm{N}, 21.47$; found, $\%$ : C, 55.21; H, 5.13; N, 21.41.

Synthesis of $\mathbf{N}$-[3-(1H-1,2,3-benzotriazol-1-yl)-propyl]urea (compound 2). The compound $1(0.273 \mathrm{~mol})$ and urea $(0.273 \mathrm{~mol})$ were stirred on a magnetic stirrer for about 4.00 hours. The completion of the reaction was monitored by TLC plates coated with silica gel-G. After the completion of the reaction the product was filtered and purified chromatographically on a silica gel packed column using chloroform : methanol $(8: 2 \mathrm{v} / \mathrm{v})$ as eluent $(150 \mathrm{ml})$. The purified product was recrystallized from ethanol to yield compound $\mathbf{2}$.

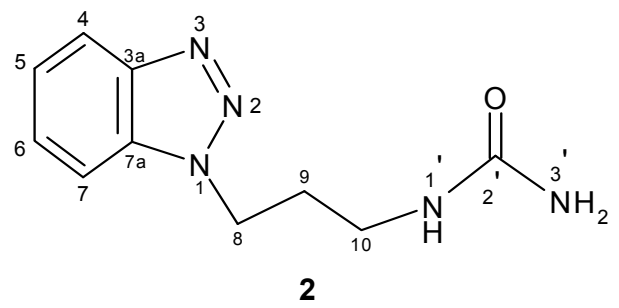

N-[3-(1H-1,2,3-benzotriazol-1-yl)propyl]urea (2). Yield: 70\%; m.p. 60$63{ }^{\circ} \mathrm{C}$; IR spectrum $\left(\mathrm{cm}^{-1}\right): 1330(\mathrm{~N}-\mathrm{C}), 1464(\mathrm{C}=\mathrm{C}), 1543(\mathrm{~N}=\mathrm{N}), 1662(\mathrm{CO})$, 1431, 2835, $2894\left(\mathrm{CH}_{2}\right), 3022(\mathrm{CH}-\mathrm{Ar}), 3365(\mathrm{NH}), 3415\left(\mathrm{NH}_{2}\right) ;{ }^{1} \mathrm{H}$ NMR spectrum (300 MHz, $\left.\mathrm{CDCl}_{3}, \mathrm{TMS}\right), \delta: 2.15-2.20(\mathrm{~m}, 2 \mathrm{H}, \mathrm{H}-9) ; 3.38-3.42(\mathrm{~m}$, 2H, H-10); 4.17 (t, 2H, J=7.40 Hz, H-8); 5.69 (s, 1H, H-1'); 5.96 (s, 2H, H-3'); 7.32-7.94 (m, 4H, Ar-H); ${ }^{13} \mathrm{C}$ NMR spectrum (75 MHz, $\left.\mathrm{CDCl}_{3}, \mathrm{TMS}\right), \delta: 35.5$ (C-9); 41.2 (C-10); 48.2 (C-8); 117.6 (C-4); 121.3 (C-7); 127.8 (C-5); 128.2 (C-

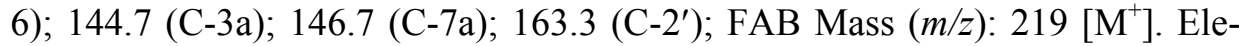
mental analysis data for $\mathrm{C}_{10} \mathrm{H}_{13} \mathrm{~N}_{5} \mathrm{O}$ : calculated, \%: $\mathrm{C}, 54.78 ; \mathrm{H}, 5.97 ; \mathrm{N}, 31.94$; found, \%: C, 54.79; H, 5.90; N, 31.88 .

Synthesis of $\mathrm{N}$-[3-(1H-1,2,3-benzotriazol-1-yl)propyl]-N'-[(phenyl)methylidene]urea (compound 3a). The compound 2 (0.036 mol) and benzaldehyde $(0.036 \mathrm{~mol})$ in methanol $(100 \mathrm{ml})$ in the presence of $2-4$ drops of glacial acetic acid were first stirred on a magnetic stirrer for about 2.00 hours at room temperature followed by reflux on a steam bath for about 3.00 hours. The completion of the reaction was monitored by TLC plates coated with silica gelG. The product was filtered, cooled and purified on silica gel using $\mathrm{CH}_{3} \mathrm{OH}$ : $\mathrm{CHCl}_{3}(7: 3 \mathrm{v} / \mathrm{v})$ system as eluent $(80 \mathrm{ml})$. The purified product was dried under vacuum and recrystallized from ethanol at room temperature to furnish the compound 3a. 


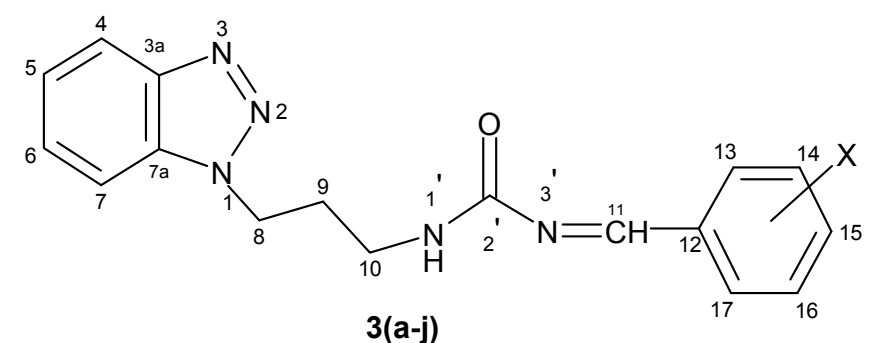

N-[3-(1H-1,2,3-benzotriazol-1-yl)propyl]-N'-[(phenyl)methylidene]urea (3a). Yield: $60 \%$; m.p. $80-83{ }^{\circ} \mathrm{C}$; IR spectrum $\left(\mathrm{cm}^{-1}\right)$ : $1301(\mathrm{~N}-\mathrm{C}), 1462(\mathrm{C}=\mathrm{C})$, $1541(\mathrm{~N}=\mathrm{N}), 1550(\mathrm{~N}=\mathrm{CH}), 1661(\mathrm{CO}), 1429,2834,2892\left(\mathrm{CH}_{2}\right), 3020(\mathrm{CH}-$ Ar), $3361(\mathrm{NH}) ;{ }^{1} \mathrm{H}$ NMR spectrum (300 MHz, $\left.\mathrm{CDCl}_{3}, \mathrm{TMS}\right), \delta: 2.04-2.09$ (m, 2H, H-9); 3.28-3.34 (m, 2H, H-10); 4.19 (t, 2H, $J=7.35 \mathrm{~Hz}, \mathrm{H}-8) ; 5.76$ (s, 1H, H-1'); 7.94 (s, 1H, H-11); 7.15-7.85 (m, 9H, Ar-H); ${ }^{13} \mathrm{C}$ NMR spectrum (75 MHz, $\mathrm{CDCl}_{3}$, TMS), $\delta$ : 38.4 (C-9); 42.3 (C-10); 47.3 (C-8); 115.3 (C-4); 120.0 (C-7); 125.8 (C-5); 126.3 (C-13 and C-17); 127.5 (C-14 and C-16); 128.5 (C-6); 129.2 (C-15); 131.2 (C-12); 136.2 (C-3a); 145.2 (C-11); 146.1 (C-7a); $162.6\left(\mathrm{C}-2^{\prime}\right)$; FAB Mass $(\mathrm{m} / \mathrm{z}): 307\left[\mathrm{M}^{+}\right]$. Elemental analysis data for $\mathrm{C}_{17} \mathrm{H}_{17} \mathrm{~N}_{5} \mathrm{O}$ : calculated, \%: C, 66.43; H, 5.57; N, 22.78; found, \%: C, 66.40; $\mathrm{H}$, $5.48 ; \mathrm{N}, 22.72$.

Compounds $3(\mathbf{b}-\mathbf{j})$ have also been synthesized using similar method.

Synthesis of $\mathbf{N}$-[3-(1H-1,2,3-benzotriazol-1-yl)propyl]-2-(phenyl)-4-oxo1,3-thiazolidine carboxamide (compound 4a). The compound 3a $(0.016 \mathrm{~mol})$ and thioglycolic acid $(0.016 \mathrm{~mol})$ in methanol $(50 \mathrm{ml})$ in the presence of $\mathrm{ZnCl}_{2}$ were first stirred on a magnetic stirrer for about 2.30 hours at room temperature followed by reflux on a steam bath for about 6.00 hours. The completion of the reaction was monitored by TLC plates coated with silica gel-G. The product was filtered, cooled and purified on a column packed with silica gel using the system $\mathrm{CH}_{3} \mathrm{OH}: \mathrm{CHCl}_{3}(7: 3 \mathrm{v} / \mathrm{v})$ as eluent $(70 \mathrm{ml})$. The purified product was dried under vacuum and recrystallized from ethanol at room temperature to furnish compound $\mathbf{4 a}$.

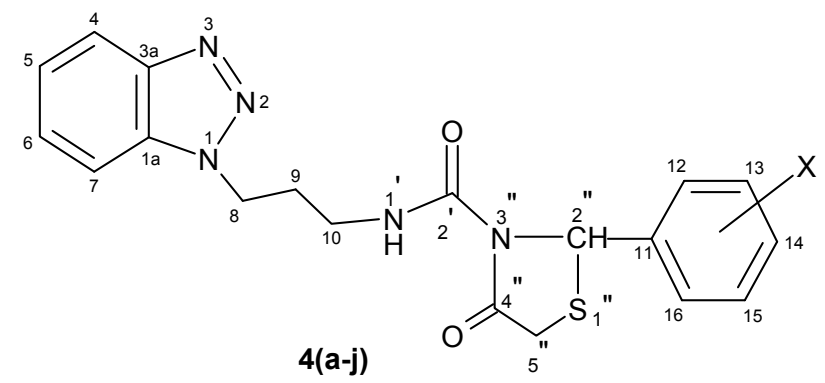

N-[3-(1H-1,2,3-benzotriazol-1-yl)propyl]-2-(phenyl)-4-oxo-1,3-thiazolidine carboxamide (4a). Yield: $62 \%$; m.p. $81-82{ }^{\circ} \mathrm{C}$; IR spectrum $\left(\mathrm{cm}^{-1}\right): 668$ $(\mathrm{C}-\mathrm{S}-\mathrm{C}), 1332(\mathrm{C}-\mathrm{N}), 1478(\mathrm{C}=\mathrm{C}), 1571(\mathrm{~N}=\mathrm{N}), 1672(\mathrm{CO}), 1740$ (CO cyclic), 1438, 2843, $2903\left(\mathrm{CH}_{2}\right), 2940\left(\mathrm{~S}-\mathrm{CH}_{2}\right), 3032(\mathrm{CH}-\mathrm{Ar}), 3373(\mathrm{NH}) ;{ }^{1} \mathrm{H}$ NMR spectrum (300 MHz, $\mathrm{CDCl}_{3}$, TMS), $\delta: 2.18-2.22$ (m, 2H, H-9); 3.37 (s, 2H, H-5"); 3.40-3.44 (m, 2H, H-10); 4.10 (t, 2H, $J=7.35 \mathrm{~Hz}, \mathrm{H}-8) ; 5.15$ (s, 1H, $\left.\mathrm{H}-2^{\prime \prime}\right) ; 5.80\left(\mathrm{~s}, 1 \mathrm{H}, \mathrm{H}-1^{\prime}\right) ; 6.72-8.09(\mathrm{~m}, 9 \mathrm{H}, \mathrm{Ar}-\mathrm{H}) ;{ }^{13} \mathrm{C}$ NMR spectrum 
(75 MHz, $\mathrm{CDCl}_{3}$, TMS), $\delta: 33.5$ (C-5"); 36.6 (C-9); 45.1 (C-10); 47.3 (C-8); $61.7\left(\mathrm{C}-2^{\prime \prime}\right) ; 110.3$ (C-4); 118.9 (C-7); 125.7 (C-5); 126.4 (C-12 and C-16); 128.4 (C-6); 129.8 (C-14); 130.1 (C-13 and C-15); 132.6 (C-3a); 136.4 (C-11); 145.9 (C-7a); 161.1 (C-2'); 168.7 (C-4"); FAB Mass ( $\mathrm{m} / \mathrm{z}): 381\left[\mathrm{M}^{+}\right]$. Elemental analysis data for $\mathrm{C}_{19} \mathrm{H}_{19} \mathrm{~N}_{5} \mathrm{O}_{2} \mathrm{~S}$ : calculated, \%: C, 59.82; H, 5.02; N, 18.35; found, \%: C, 59.74; $\mathrm{H}, 4.97 ; \mathrm{N}, 18.29$.

Compounds $4(\mathbf{b}-\mathbf{j})$ have also been synthesized using the similar method.

Synthesis of N-[3-(1H-1,2,3-benzotriazol-1-yl)propyl]-2-(phenyl)-4-oxo5-(benzylidene)-1,3-thiazolidine carboxamides (compound 5a). The compound $4 \mathrm{a}(0.010 \mathrm{~mol})$ and benzaldehyde $(0.010 \mathrm{~mol})$ in methanol $(50 \mathrm{ml})$ in the presence of $\mathrm{CH}_{3} \mathrm{CH}_{2} \mathrm{ONa}$ were first stirred on a magnetic stirrer for about 2.00 hours at room temperature followed by reflux on a steam bath for about 4.00 hours. The completion of the reaction was monitored by TLC plates coated with silica gel-G. The product was filtered, cooled and purified on silica gel using the system $\mathrm{CH}_{3} \mathrm{OH}: \mathrm{CHCl}_{3}(7: 3 \mathrm{v} / \mathrm{v})$ as eluent $(90 \mathrm{ml})$. The purified product was dried under vacuum and recrystallized from ethanol at room temperature to furnish compound $\mathbf{5 a}$.

Compounds 5 (b-j) have also been synthesized by using the similar method.

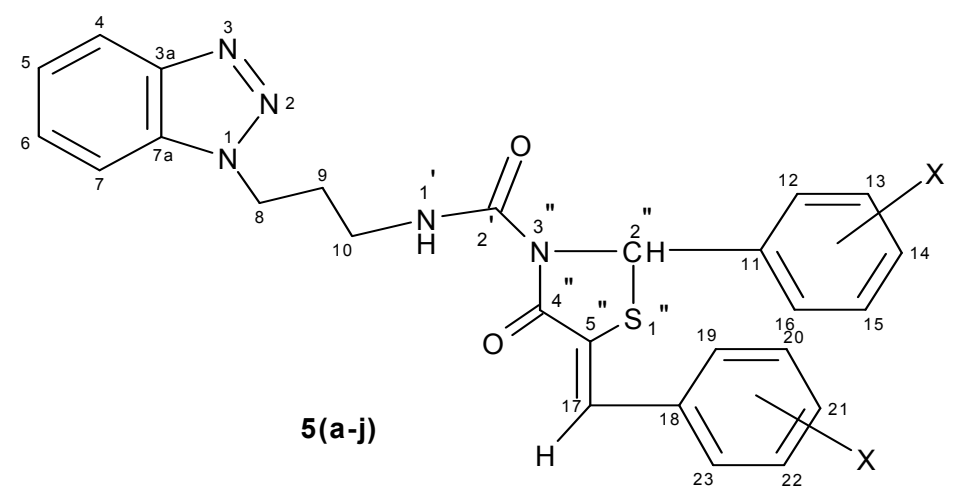

N-[3-(1H-1,2,3-benzotriazol-1-yl)propyl]-2-(phenyl)-4-oxo-5-(benzylidene)-1,3-thiazolidine carboxamide (5a). Yield: 57\%; m.p. $79-81{ }^{\circ} \mathrm{C}$; IR spectrum $\left(\mathrm{cm}^{-1}\right): 1560(\mathrm{C}=\mathrm{CH}), 1336(\mathrm{C}-\mathrm{N}), 1477(\mathrm{C}=\mathrm{C}), 1544(\mathrm{~N}=\mathrm{N}), 1674$ (CO), 1737 (CO cyclic), 1445, 2842, $2903\left(\mathrm{CH}_{2}\right), 3034(\mathrm{CH}-\mathrm{Ar}), 3375(\mathrm{NH})$, $2850(\mathrm{C}=\mathrm{CH}), 3339(\mathrm{NH}) ;{ }^{1} \mathrm{H}$ NMR spectrum (300 MHz, $\left.\mathrm{CDCl}_{3}, \mathrm{TMS}\right), \delta$ : 2.24-2.29 (m, 2H, H-9); 3.48-3.53 (m, 2H, H-10); 4.37 (t, $2 \mathrm{H}, J=7.50 \mathrm{~Hz}$, H-8); 5.72 (s, 1H, H-1'); 5.27(s, 1H, H-2"'); $6.52(\mathrm{H}-17) ; 6.86-7.72(\mathrm{~m}, 14 \mathrm{H}$, $\mathrm{Ar}-\mathrm{H}) ;{ }^{13} \mathrm{C}$ NMR spectrum (75 MHz, $\left.\mathrm{CDCl}_{3}, \mathrm{TMS}\right), \delta: 38.2$ (C-9); 45.2 (C-10); 51.1 (C-8); 63.7 (C-2"); 110.3 (C-4); 118.9 (C-7); 125.7 (C-5); 126.4 (C-12 and C-16); 126.4 (C-19 and C-23); 128.4 (C-6); 129.8 (C-16); 129.8 (C-14); 130.1 (C-13 and C-15); 131.7 (C-20 and C-22); 132.6 (C-3a); 136.4 (C-11); 138.7 (C-18); 136.7 (C-17); 143.2 (C-5"'); 145.9 (C-7a); 161.1 (C-2'); $168.7\left(\mathrm{C}-4^{\prime \prime}\right)$; FAB Mass $(\mathrm{m} / \mathrm{z})$ : $469\left[\mathrm{M}^{+}\right]$. Elemental analysis data for $\mathrm{C}_{26} \mathrm{H}_{23} \mathrm{~N}_{5} \mathrm{O}_{2} \mathrm{~S}$ : calculated, \%: C, 66.50; H, 4.93; N, 14.91; found, \%: $\mathrm{C}, 66.46 ; \mathrm{H}$, $4.89 ; \mathrm{N}, 14.87$.

N-[3-(1H-1,2,3-benzotriazol-1-yl)propyl]-2-(4-chlorophenyl)-4-oxo-5-(4chlorobenzylidene)-1,3-thiazolidine carboxamide (5b). Yield: 60\%; m.p. 89$91^{\circ} \mathrm{C}$; IR spectrum $\left(\mathrm{cm}^{-1}\right): 732(\mathrm{C}-\mathrm{Cl}), 1470(\mathrm{C}=\mathrm{CH}), 2868(\mathrm{C}=\mathrm{CH}), 1344(\mathrm{C}-$ 
$\mathrm{NH}), 1485(\mathrm{C}=\mathrm{C}), 1546(\mathrm{~N}=\mathrm{N}), 1680(\mathrm{CO}), 1742$ (CO cyclic), 1452, 2846, $2908\left(\mathrm{CH}_{2}\right), 3039(\mathrm{CH}-\mathrm{Ar}), 3381(\mathrm{NH}) ;{ }^{1} \mathrm{H}$ NMR spectrum $\left(300 \mathrm{MHz}, \mathrm{CDCl}_{3}\right.$, TMS), $\delta: 2.41-2.46(\mathrm{~m}, 2 \mathrm{H}, \mathrm{H}-9) ; 3.59-3.63(\mathrm{~m}, 2 \mathrm{H}, \mathrm{H}-10) ; 4.50$ (t, $2 \mathrm{H}, J=$ $7.45 \mathrm{~Hz}, \mathrm{H}-8)$; 5.79 (s, 1H, H-1'); 5.21 (s, 1H, H-2"'); 6.71 (s, 1H, H-17); 6.86$7.72(\mathrm{~m}, 12 \mathrm{H}, \mathrm{Ar}-\mathrm{H}) ;{ }^{13} \mathrm{C}$ NMR spectrum (75 MHz, $\left.\mathrm{CDCl}_{3}, \mathrm{TMS}\right), \delta: 39.7$ (C-9); 42.9 (C-10); 50.4 (C-8); 62.2 (C-2"); 116.2 (C-4); 120.9 (C-7); 123.7 (C-5); 127.7 (C-12 and C-16); 128.0 (C-19 and C-23); 128.6 (C-6); 129.4 (C-13 and C-15); 130.3 (C-20 and C-22); 131.4 (C-16); 131.9 (C-14); 132.8 (C-3a); 136.7 (C-18); 136.7 (C-11); 137.2 (C-17); 143.5 (C-5"); 146.9 (C-7a); 164.1 $\left(\mathrm{C}-2^{\prime}\right) ; 174.5\left(\mathrm{C}-4^{\prime \prime}\right)$; Mass FAB $(\mathrm{m} / \mathrm{z})$ : $538\left[\mathrm{M}^{+}\right]$. Elemental analysis data for $\mathrm{C}_{26} \mathrm{H}_{21} \mathrm{~N}_{5} \mathrm{O}_{2} \mathrm{SCl}_{2}$ : calculated, \%: C, 57.99; $\mathrm{H}, 3.93 ; \mathrm{N}, 13.00$; found, \%: $\mathrm{C}$, $57.94 ; \mathrm{H}, 3.87$; N, 12.94.

N-[3-(1H-1,2,3-benzotriazol-1-yl)propyl]-2-(3-chlorophenyl)-4-oxo-5-(3chlorobenzylidene)-1,3-thiazolidine carboxamide (5c). Yield: 62\%; m.p. 86$87{ }^{\circ} \mathrm{C}$; IR spectrum $\left(\mathrm{cm}^{-1}\right): 738(\mathrm{C}-\mathrm{Cl}), 1335(\mathrm{~N}-\mathrm{C}), 1474(\mathrm{C}=\mathrm{CH}), 2862$ $(\mathrm{C}=\mathrm{CH}) 1489(\mathrm{C}=\mathrm{C}), 1552(\mathrm{~N}=\mathrm{N}), 1685(\mathrm{CO}), 1747$ (CO cyclic), 1452, 2854, $2914\left(\mathrm{CH}_{2}\right), 3047(\mathrm{CH}-\mathrm{Ar}), 3382(\mathrm{NH}) ;{ }^{1} \mathrm{H}$ NMR spectrum $\left(300 \mathrm{MHz}, \mathrm{CDCl}_{3}\right.$, TMS), $\delta: 2.45-2.49$ (m, 2H, H-9); 3.60-3.65 (m, 2H, H-10); 4.47 (t, 2H, $J=$ $7.50 \mathrm{~Hz}, \mathrm{H}-8) ; 5.95$ (s, 1H, H-1'); 5.23 (s, 1H, H-2"'); 6.68 (s, 1H, H-17); 6.86$7.72(\mathrm{~m}, 12 \mathrm{H}, \mathrm{Ar}-\mathrm{H}) ;{ }^{13} \mathrm{C}$ NMR spectrum $\left(75 \mathrm{MHz}, \mathrm{CDCl}_{3}, \mathrm{TMS}\right), \delta: 38.6$ (C-9); 43.6 (C-10); 47.3 (C-8); 61.9 (C-2"); 114.2 (C-4); 118.4 (C-7); 124.3 (C-5); 126.7 (C-12); 127.5 (C-19); 128.3 (C-16); 128.9 (C-23); 129.1 (C-6); 129.9 (C-14); 130.5 (C-21); 131.4 (C-13); 132.9 (C-22); 134.4 (C-3a); 135.3 (C-13); 136.6 (C-20); 138.1 (C-11); 139.7 (C-18); 140.1 (C-17); 143.2 (C-5");

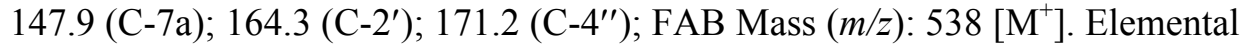
analysis data for $\mathrm{C}_{26} \mathrm{H}_{21} \mathrm{~N}_{5} \mathrm{O}_{2} \mathrm{SCl}_{2}$ : calculated, \%: $\mathrm{C}, 57.99 ; \mathrm{H}, 3.93 ; \mathrm{N}, 13.00$; found, \%: C, 57.92; H, 3.76; N, 12.91 .

N-[3-(1H-1,2,3-benzotriazol-1-yl)propyl]-2-(2-chlorophenyl)-4-oxo-5-(2chlorobenzylidene)-1,3-thiazolidine carboxamide (5d). Yield: 61\%; m.p. 82$83{ }^{\circ} \mathrm{C}$; IR spectrum $\left(\mathrm{cm}^{-1}\right): 742(\mathrm{C}-\mathrm{Cl}), 1576(\mathrm{C}=\mathrm{CH}), 2866(\mathrm{C}=\mathrm{CH}) 1338(\mathrm{C}-$ $\mathrm{NH}), 1492(\mathrm{C}=\mathrm{C}), 1549(\mathrm{~N}=\mathrm{N}), 1684(\mathrm{CO}), 1752$ (CO cyclic), 1454, 2857, $2917\left(\mathrm{CH}_{2}\right), 3046(\mathrm{CH}-\mathrm{Ar}), 3388(\mathrm{NH}) ;{ }^{1} \mathrm{H}$ NMR spectrum $\left(300 \mathrm{MHz}, \mathrm{CDCl}_{3}\right.$, TMS), $\delta: ~ 2.37-2.43$ (m, 2H, H-9); 3.53-3.59 (m, 2H, H-10); 4.44 (t, 2H, $J=$ $7.55 \mathrm{~Hz}, \mathrm{H}-8) ; 5.85$ (s, 1H, H-1'); 5.29 (s, 1H, H-2"'); 6.72 (s, 1H, H-17); 6.86$7.72(\mathrm{~m}, 12 \mathrm{H}, \mathrm{Ar}-\mathrm{H}) ;{ }^{13} \mathrm{C}$ NMR spectrum $\left(75 \mathrm{MHz}, \mathrm{CDCl}_{3}, \mathrm{TMS}\right), \delta: 36.8$ (C-9); 43.8 (C-10); 47.6 (C-8); 63.4 (C-2"); 114.5 (C-4); 119.9 (C-7); 124.7 (C5); 127.6 (C-13); 128.2 (C-22); 128.9 (C-6); 129.4 (C-13); 130.1 (C-20); 130.4 (C-14); 131.6 (C-21); 132.2 (C-16); 132.2 (C-23); 133.3 (C-3a); 135.1 (C-12); 135.1 (C-19); 137.9 (C-11); 137.9 (C-8); 138.3 (C-17); 143.1 (C-5”); 147.4

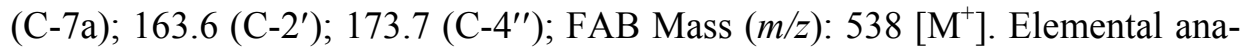
lysis data for $\mathrm{C}_{26} \mathrm{H}_{21} \mathrm{~N}_{5} \mathrm{O}_{2} \mathrm{SCl}_{2}$ : calculated, \%: C, 57.99; H, 3.93; N, 13.00; found, \%: C, 57.90; H; 3.85, N, 12.90 .

N-[3-(1H-1,2,3-benzotriazol-1-yl)-propyl]-2-(4-bromophenyl)-4-oxo-5(4-bromobenzylidene)-1,3-thiazolidine carboxamide (5e). Yield: 62\%; m.p. 78-79 ${ }^{\circ} \mathrm{C}$; IR spectrum $\left(\mathrm{cm}^{-1}\right): 561(\mathrm{C}-\mathrm{Br}), 1472(\mathrm{C}=\mathrm{CH}), 2856(\mathrm{C}=\mathrm{CH}) 1346$ $(\mathrm{C}-\mathrm{N}), 1490(\mathrm{C}=\mathrm{C}), 1553(\mathrm{~N}=\mathrm{N}), 1681(\mathrm{CO}), 1750$ (CO cyclic), 1455, 2852, $2915\left(\mathrm{CH}_{2}\right), 3042(\mathrm{CH}-\mathrm{Ar}), 3385(\mathrm{NH}) ;{ }^{1} \mathrm{H}$ NMR spectrum $\left(300 \mathrm{MHz}, \mathrm{CDCl}_{3}\right.$, TMS), $\delta: 2.38-2.42$ (m, 2H, H-9); 3.52-3.57 (m, 2H, H-10); 4.46 (t, 2H, $J=$ $7.45 \mathrm{~Hz}, \mathrm{H}-8) ; 5.92$ (s, 1H, H-1'); 5.11 (s, 1H, H-2' ); 6.77 (s, 1H, H-17); 6.86- 
$7.72(\mathrm{~m}, 12 \mathrm{H}, \mathrm{Ar}-\mathrm{H}) ;{ }^{13} \mathrm{C}$ NMR spectrum $\left(75 \mathrm{MHz}, \mathrm{CDCl}_{3}, \mathrm{TMS}\right), \delta: 39.4$ (C-9); 43.8 (C-10); 47.6 (C-8); 63.4 (C-2"); 112.4 (C-4); 119.4 (C-7); 123.9 (C-14); 123.9 (C-21); 124.5 (C-5); 128.6 (C-6); 130.8 (C-12 and C-16); 131.2 (C-19 and C-23); 131.4 (C-13 and C-15); 131.8 (C-20 and C-22); 132.6 (C-3a); 136.5 (C-11); 137.7 (C-18); 138.8 (C-17); 141.9 (C-5"); 147.9 (C-7a); 164.2 (C$\left.2^{\prime}\right) ; 172.3\left(\mathrm{C}-4^{\prime \prime}\right)$; FAB Mass $(\mathrm{m} / \mathrm{z}): 627\left[\mathrm{M}^{+}\right]$. Elemental analysis data for $\mathrm{C}_{26} \mathrm{H}_{21} \mathrm{~N}_{5} \mathrm{O}_{2} \mathrm{SBr}_{2}$ : calculated, \%: C, 49.77; $\mathrm{H}, 3.37 ; \mathrm{N}, 11.16$; found, \%: $\mathrm{C}$, $49.73 ; \mathrm{H}, 3.33 ; \mathrm{N}, 11.12$.

N-[3-(1H-1,2,3-benzotriazol-1-yl)propyl]-2-(3-bromophenyl)-4-oxo-5-(3bromobenzylidene)-1,3-thiazolidine carboxamide (5f). Yield: 64\%; m.p. 81$82{ }^{\circ} \mathrm{C}$; IR spectrum $\left(\mathrm{cm}^{-1}\right): 568(\mathrm{C}-\mathrm{Br}), 2852(\mathrm{C}=\mathrm{CH}), 1475(\mathrm{C}=\mathrm{CH}), 1347$ $(\mathrm{C}-\mathrm{NH}), 1484(\mathrm{C}=\mathrm{C}), 1550(\mathrm{~N}=\mathrm{N}), 1679(\mathrm{CO}), 1745$ (CO cyclic), 1450, 2848, $2909\left(\mathrm{CH}_{2}\right), 3040(\mathrm{CH}-\mathrm{Ar}), 3384(\mathrm{NH}) ;{ }^{1} \mathrm{H}$ NMR spectrum $\left(300 \mathrm{MHz}, \mathrm{CDCl}_{3}\right.$, TMS), $\delta$ : 2.40-2.44 (m, 2H, H-9); 3.60-3.67 (m, 2H, H-10); 4.51 (t, $2 \mathrm{H}, J=$ $7.40 \mathrm{~Hz}, \mathrm{H}-8) ; 5.89$ (s, 1H, H-1'); 5.19 (s, 1H, H-2"'); 6.68 (s, 1H, H-17); 6.86$7.72(\mathrm{~m}, 12 \mathrm{H}, \mathrm{Ar}-\mathrm{H}) ;{ }^{13} \mathrm{C}$ NMR spectrum $\left(75 \mathrm{MHz}, \mathrm{CDCl}_{3}, \mathrm{TMS}\right), \delta: 37.5(\mathrm{C}-$ 9); 44.3 (C-10); 49.3 (C-8); 64.6 (C-2"); 109.2 (C-4); 118.9 (C-7); 123.7 (C13); 124.6 (C-20); 124.7 (C-5); 125.6 (C-16); 125.6 (C-23); 128.4 (C-6); 129.8 (C-12); 130.8 (C-19); 132.5 (C-13); 133.4 (C-22); 133.9 (C-14); 134.3 (C-21); 134.5 (C-3a); 140.3 (C-11); 140.8 (C-17); 142.5 (C-5"); 142.7 (C-18); 145.6 (C-7a); $163.7\left(\mathrm{C}-2^{\prime}\right) ; 172.6\left(\mathrm{C}-4^{\prime \prime}\right)$; FAB Mass $(\mathrm{m} / \mathrm{z})$ : $627\left[\mathrm{M}^{+}\right]$. Elemental analysis data for $\mathrm{C}_{26} \mathrm{H}_{21} \mathrm{~N}_{5} \mathrm{O}_{2} \mathrm{SBr}_{2}$ : calculated, \%: C, 49.77; $\mathrm{H}, 3.37 ; \mathrm{N}, 11.16$; found, \%: C, 49.72; H, 3.34; N, 11.14.

N-[3-(1H-1,2,3-benzotriazol-1-yl)-propyl]-2-(2-bromophenyl)-4-oxo-5-(2bromobenzylidene)-1,3-thiazolidine carboxamide (5g). Yield: 63\%; m.p. 75$76{ }^{\circ} \mathrm{C}$; IR spectrum $\left(\mathrm{cm}^{-1}\right): 564(\mathrm{C}-\mathrm{Br}), 1479(\mathrm{C}=\mathrm{CH}), 2856(\mathrm{C}=\mathrm{CH}), 1352$ $(\mathrm{C}-\mathrm{NH}), 1494(\mathrm{C}=\mathrm{C}), 1551(\mathrm{~N}=\mathrm{N}), 1686(\mathrm{CO}), 1749$ (CO cyclic), 1456, 2857, $2915\left(\mathrm{CH}_{2}\right), 3045(\mathrm{CH}-\mathrm{Ar}), 3386(\mathrm{NH}) ;{ }^{1} \mathrm{H}$ NMR spectrum $\left(300 \mathrm{MHz}, \mathrm{CDCl}_{3}\right.$, TMS), $\delta: ~ 2.40-2.45$ (m, 2H, H-9); 3.55-3.61 (m, 2H, H-10); 4.49 (t, 2H, $J=$ $7.50 \mathrm{~Hz}, \mathrm{H}-8)$; 5.91 (s, 1H, H-1'); 5.17 (s, 1H, H-2' ); 6.59 (s, 1H, H-17); 6.86$7.72(\mathrm{~m}, 12 \mathrm{H}, \mathrm{Ar}-\mathrm{H}) ;{ }^{13} \mathrm{C}$ NMR spectrum $\left(75 \mathrm{MHz}, \mathrm{CDCl}_{3}, \mathrm{TMS}\right), \delta: 40.3$ (C-9); 45.9 (C-10); 48.4 (C-8); 62.6 (C-2"); 111.1 (C-4); 119.5 (C-7); 120.3 (C-12); 122.6 (C-19); 125.7 (C-5); 126.5 (C-15); 127.2 (C-22); 128.4 (C-6); 129.3 (C-16); 130.1 (C-23); 131.3 (C-14); 132.8 (C-21); 133.2 (C-3a); 134.5 (C-13); 135.7 (C-20); 138.7 (C-17); 142.2 (C-5"); 142.6 (C-11); 144.2 (C-22); 147.9 (C-7a); $163.1\left(\mathrm{C}-2^{\prime}\right) ; 172.5\left(\mathrm{C}-4^{\prime \prime}\right) ;$ FAB Mass $(\mathrm{m} / \mathrm{z}): 627\left[\mathrm{M}^{+}\right]$. Elemental analysis data for $\mathrm{C}_{26} \mathrm{H}_{21} \mathrm{~N}_{5} \mathrm{O}_{2} \mathrm{SBr}_{2}$ : calculated, \%: C, 49.77; $\mathrm{H}, 3.37 ; \mathrm{N}, 11.16$; found, \%: C, 49.70; H, 3.30; N, 11.10.

N-[3-(1H-1,2,3-benzotriazol-1-yl)propyl]-2-(4-nitrophenyl)-4-oxo-5-(4nitrobenzylidene)-1,3-thiazolidine carboxamide (5h). Yield: $60 \%$; m.p. $73-$ $74{ }^{\circ} \mathrm{C}$; IR spectrum $\left(\mathrm{cm}^{-1}\right): 865(\mathrm{C}-\mathrm{NO}), 1495(\mathrm{~N}=\mathrm{O}), 1578(\mathrm{C}=\mathrm{CH}), 2857$ $(\mathrm{C}=\mathrm{CH}), 1345(\mathrm{C}-\mathrm{N}), 1488(\mathrm{C}=\mathrm{C}), 1547(\mathrm{~N}=\mathrm{N}), 1683(\mathrm{CO}), 1744(\mathrm{CO}$ cyclic $)$, 1451, 2850, $2912\left(\mathrm{CH}_{2}\right), 3045(\mathrm{CH}-\mathrm{Ar}), 3387(\mathrm{NH}) ;{ }^{1} \mathrm{H}$ NMR spectrum (300 MHz, $\mathrm{CDCl}_{3}, \mathrm{TMS}$ ), $\delta:$ 2.34-2.39 (m, 2H, H-9); 3.52-3.58 (m, 2H, H-10); $4.50(\mathrm{t}, 2 \mathrm{H}, J=7.55 \mathrm{~Hz}, \mathrm{H}-8) ; 5.13\left(\mathrm{~s}, 1 \mathrm{H}, \mathrm{H}-2^{\prime \prime}\right) ; 5.87$ (s, 1H, H-1'); 6.61 (s, 1H, H-17); 6.81-7.71 (m,12H, Ar-H); ${ }^{13} \mathrm{C} \mathrm{NMR} \mathrm{spectrum}\left(75 \mathrm{MHz}, \mathrm{CDCl}_{3}\right.$, TMS), $\delta$ : 37.7 (C-9); 44.8 (C-10); 48.9 (C-8); 64.2 (C-2"); 112.2 (C-4); 118.5 (C-7); 122.6 (C-13 and C-15); 123.2 (C-20 and C-22); 124.8 (C-5); 126.9 (C-12 and $\mathrm{C}-16) ; 127.4$ (C-19 and C-23); 128.3 (C-6); 132.4 (C-3a); 139.8 (C-11); 
138.3 (C-17); 140.3 (C-18); 142.3 (C-5"); 145.9 (C-7a); 147.9 (C-14); 148.5

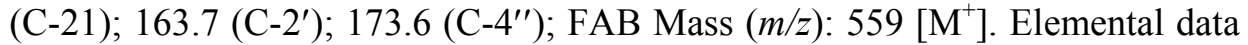
analysis for $\mathrm{C}_{26} \mathrm{H}_{21} \mathrm{~N}_{7} \mathrm{O}_{6} \mathrm{~S}$ : calculated, $\%$ : C, $55.80 ; \mathrm{H}, 3.78 ; \mathrm{N}, 17.52$; found, \%: C, 55.77; H, 3.72; N, 17.48.

N-[3-(1H-1,2,3-benzotriazol-1-yl)propyl]-2-(3-nitrophenyl)-4-oxo-5-(3nitrobenzylidene)-1,3-thiazolidine carboxamide (5i). Yield: 64\%; m.p. 81$82{ }^{\circ} \mathrm{C}$; IR spectrum $\left(\mathrm{cm}^{-1}\right): 841(\mathrm{C}-\mathrm{NO}), 1480(\mathrm{C}=\mathrm{CH}), 1497(\mathrm{~N}=\mathrm{O}), 2864$ $(\mathrm{C}=\mathrm{CH}), 1350(\mathrm{C}-\mathrm{N}), 1493(\mathrm{C}=\mathrm{C}), 1548(\mathrm{~N}=\mathrm{N}), 1682(\mathrm{CO}), 1748(\mathrm{CO}$ cyclic $)$, 1453, 2853, $2910\left(\mathrm{CH}_{2}\right), 3044(\mathrm{CH}-\mathrm{Ar}), 3384(\mathrm{NH}) ;{ }^{1} \mathrm{H}$ NMR spectrum (300 MHz, $\left.\mathrm{CDCl}_{3}, \mathrm{TMS}\right), \delta: 2.43-2.48$ (m, 2H, H-9); 3.50-3.54 (m, 2H, H-10); 4.48 (t, 2H, $J=7.50 \mathrm{~Hz}, \mathrm{H}-8) ; 5.15$ (s, 1H, H-2"); 5.90 (s, 1H, H-1'); 6.69 (s, $1 \mathrm{H}, \mathrm{H}-17) ; 6.79-7.68(\mathrm{~m}, 12 \mathrm{H}, \mathrm{Ar}-\mathrm{H}) ;{ }^{13} \mathrm{C} \mathrm{NMR}$ spectrum $\left(75 \mathrm{MHz}, \mathrm{CDCl}_{3}\right.$, TMS), $\delta$ : 40.2 (C-9); 45.2 (C-10); 49.7 (C-8); 65.8 (C-2"); 113.3 (C-4); 118.9 (C-7); 121.8 (C-12); 122.7 (C-19); 123.6 (C-14); 124.8 (C-21); 125.9 (C-5); 128.8 (C-6); 129.4 (C-15); 130.4 (C-22); 132.6 (C-3a); 132.9 (C-16); 134.2 (C-23); 139.7 (C-11); 140.3 (C-17); 141.4 (C-18); 143.1 (C-5"); 146.9 (C-7a); 147.9 (C-13); 148.2 (C-20); 163.1 (C-2'); $175.6\left(\mathrm{C}-4^{\prime \prime}\right)$; FAB Mass $(\mathrm{m} / \mathrm{z}): 559$ $\left[\mathrm{M}^{+}\right]$. Elemental analysis data for $\mathrm{C}_{26} \mathrm{H}_{21} \mathrm{~N}_{7} \mathrm{O}_{6} \mathrm{~S}$ : calculated, \%: C, 55.80; $\mathrm{H}$, 3.78; N, 17.52; found, \%: C, 55.75; H, 3.73; N, 17.46 .

N-[3-(1H-1,2,3-benzotriazol-1-yl)propyl]-2-(2-nitrophenyl)-4-oxo-5-(2nitrobenzylidene)-1,3-thiazolidine carboxamide (5j). Yield: 62\%; m.p. 84$85{ }^{\circ} \mathrm{C}$; IR spectrum $\left(\mathrm{cm}^{-1}\right): 852(\mathrm{C}-\mathrm{NO}), 1478(\mathrm{~N}=\mathrm{O}), 1489(\mathrm{C}=\mathrm{CH}), 2870$ $(\mathrm{C}=\mathrm{CH}), 1355(\mathrm{C}-\mathrm{NH}), 1497(\mathrm{C}=\mathrm{C}), 1553(\mathrm{~N}=\mathrm{N}), 1689(\mathrm{CO}), 1751(\mathrm{CO}$ cyclic), 1457, 2858, $2919\left(\mathrm{CH}_{2}\right), 3047(\mathrm{CH}-\mathrm{Ar}), 3387(\mathrm{NH}) ;{ }^{1} \mathrm{H}$ NMR spectrum (300 MHz, $\mathrm{CDCl}_{3}$, TMS), $\delta: 2.34-2.40$ (m, 2H, H-9); 3.60-3.65 (m, 2H, H-10); 4.53 (t, 2H, $J=7.45 \mathrm{~Hz}, \mathrm{H}-8) ; 5.14$ (s, $\left.1 \mathrm{H}, \mathrm{H}-2^{\prime \prime}\right) ; 5.83$ (s, 1H, H-1'); 6.62 (s, $1 \mathrm{H}, \mathrm{H}-17) ; 6.89-7.75(\mathrm{~m}, 12 \mathrm{H}, \mathrm{Ar}-\mathrm{H}) ;{ }^{13} \mathrm{C}$ NMR spectrum $\left(75 \mathrm{MHz}, \mathrm{CDCl}_{3}\right.$, TMS), $\delta$ : 38.6 (C-9); 45.5 (C-10); 50.9 (C-8); 63.8 (C-2"); 112.4 (C-4); 117.4 (C-7); 121.2 (C-13); 122.5 (C-20); 123.8 (C-5); 126.8 (C-16); 127.6 (C-23); 128.6 (C-6); 130.8 (C-14); 131.3 (C-21); 132.9 (C-3a); 133.5 (C-11); 134.8 (C-18); 135.3 (C-15); 136.7 (C-22); 141.2 (C-17); 144.9 (C-5"); 145.7 (C-7a); 146.5 (C-12); 147.9 (C-19); 161.1 (C-2'); 174.5 (C-4"'); FAB Mass $(\mathrm{m} / \mathrm{z}): 559$ $\left[\mathrm{M}^{+}\right]$. Elemental analysis data for $\mathrm{C}_{26} \mathrm{H}_{21} \mathrm{~N}_{7} \mathrm{O}_{6} \mathrm{~S}$ : calculated, \%: $\mathrm{C}, 55.80 ; \mathrm{H}$, $3.78 ; \mathrm{N}, 17.52$; found, \%: C, 55.72; H, 3.74; N, 17.45 .

\section{RESULTS AND DISCUSSION}

The reaction of 1-bromo-3-chloropropane with 1,2,3-benzotriazole was carried out in methanol as solvent to afford the compound 1. The spectroscopic analyses of the compound $\mathbf{1}$ showed the occurrence of absorption peaks for $\mathrm{N}-$ $\mathrm{CH}$ and $\mathrm{C}-\mathrm{Cl}$ at $1324 \mathrm{~cm}^{-1}$ and $749 \mathrm{~cm}^{-1}$ in the IR spectrum. The IR spectrum confirms the formation of compound $\mathbf{1}$. This fact is also supported by the disappearance of $\mathrm{NH}$ absorption band of 1,2,3-benzotriazole. The compound $\mathbf{1}$ on the reaction with urea on continuous stirring at room temperature yielded compound 2. When analyzing compound $\mathbf{2}$ spectroscopically, we found three absorption peaks in IR spectrum for $\mathrm{NH}, \mathrm{NH}_{2}$ and $\mathrm{CO}$ at $3365,3415 \mathrm{~cm}^{-1}$ and $1662 \mathrm{~cm}^{-1}$, respectively, while absorption of $\mathrm{C}-\mathrm{Cl}$ had disappeared. This clearly 
indicated the formation of the compound 2. This fact was also supported by ${ }^{1} \mathrm{H}$ and ${ }^{13} \mathrm{C}$ NMR spectral data because two signals appeared in the ${ }^{1} \mathrm{H}$ NMR spectrum for $\mathrm{NH}$ and $\mathrm{NH}_{2}$ group absorption at $\delta 5.69$ and $\delta 5.96 \mathrm{ppm}$, respectively. The formation of the compound $\mathbf{2}$ was fully supported by a $\mathrm{CO}$ group signal at $\delta 163.3 \mathrm{ppm}$ in the ${ }^{13} \mathrm{C}$ NMR spectrum. All these facts together gave the strong evidence for the synthesis of compound 2 . Substituted benzaldehydes entered the condensation reaction with the compound $\mathbf{2}$ giving Schiff bases, that was confirmed by IR, ${ }^{1} \mathrm{H}$ NMR and ${ }^{13} \mathrm{C}$ NMR spectral data of compounds $\mathbf{3 a}$. In the IR spectra an absorption band was found at $1550 \mathrm{~cm}^{-1}$ while a strong signal appeared at $\delta 7.94$ and $\delta 145.2 \mathrm{ppm}$ in the ${ }^{1} \mathrm{H}$ NMR and ${ }^{13} \mathrm{C}$ NMR spectra of compounds 3a, respectively. These facts are also supported by the disappearance of the signal of $\mathrm{NH}_{2}$ group in the ${ }^{1} \mathrm{H}$ NMR spectrum of the compound 2. The compounds $\mathbf{3}(\mathbf{a}-\mathbf{j})$ on reaction with equimolar amount of thioglycolic acid in the presence of $\mathrm{ZnCl}_{2}$ (acted as a catalyst) in trace amounts gave the cycloaddition products $-\mathrm{a}$ five-membered thiazolidinone ring containing compounds, compounds $4(\mathbf{a}-\mathbf{j})$. The compounds $4 \mathbf{a}$ showed a characteristic absorption band of the cyclic carbonyl group at $1672 \mathrm{~cm}^{-1}$ in the IR spectra. The ${ }^{1} \mathrm{H}$ NMR spectra of compounds $\mathbf{4 a}$ aroused our attention and clearly indicated the presence of the active methylene protons of the thiazolidine ring at $\delta 3.37 \mathrm{ppm}$. The ${ }^{13} \mathrm{C}$ NMR spectra of compounds $4 \mathbf{a}$ also supported the fact that cyclic carbonyl group presented a signal at $\delta 168.7 \mathrm{ppm}$. All these facts were also supported by the two evidences - the disappearance of $\mathrm{N}=\mathrm{CH}$ proton and appearance of $\mathrm{N}-\mathrm{CH}$ proton at $\delta 5.15 \mathrm{ppm}$ in the ${ }^{1} \mathrm{H}$ NMR spectra of compounds $4 \mathbf{a}$. The compounds $\mathbf{4}(\mathbf{a}-\mathbf{j})$ underwent the Knoevenagel condensation reaction with substituted benzaldehydes in the presence of alkali metal alkoxide $\left(\mathrm{C}_{2} \mathrm{H}_{5} \mathrm{ONa}\right)$. We had followed the above-described procedure to afford compounds $\mathbf{5}(\mathbf{a}-\mathbf{j})$. In the ${ }^{1} \mathrm{H}$ NMR spectra of the compounds $\mathbf{5}(\mathbf{a}-\mathbf{j})$, we observed the disappearance of two methylene protons of compounds $4(\mathbf{a}-\mathbf{j})$, an appearance of a new signal for $\mathrm{C}=\mathrm{CH}$ in the range of $\delta 6.52-6.77 \mathrm{ppm}$ in the ${ }^{1} \mathrm{H}$ NMR spectra and that of two new signals for $\mathrm{C}=\mathrm{CH}$ and $\mathrm{C}=\mathrm{CH}$ groups in the range of $\delta 136.7-141.2$ and $\delta 141.9-144.9 \mathrm{ppm}$, respectively, in the ${ }^{13} \mathrm{C}$ NMR spectra of the compounds $\mathbf{5}(\mathbf{a}-\mathbf{j})$. All these facts clearly confirmed the synthesis of the final products.

The results of the all described activities (antibacterial, antifungal, antitubercular and antiinflammatory) were summarized in Tables 1 and 2. The results of the antimicrobial screening revealed that the compounds $\mathbf{5}(\mathbf{a}-\mathbf{j})$ showed considerable and varied activity against the selected microorganisms. A new series of N-[3-(1H-1,2,3-benzotriazol-1-yl)propyl]-2-(substituted phenyl)-4oxo-5-(substituted benzylidene)-1,3-thiazolidine carboxamide, compounds 5(aj), was synthesized and screened for their antimicrobial, antitubercular and antiinflammatory activity (as shown in Table 1 and 2). The results from the Tables revealed that all the synthesized compounds $\mathbf{5}(\mathbf{a}-\mathbf{j})$ possessed a structure activity relationship (SAR) because activities of compounds varied with substitution. Compounds containing nitro group (5h, $\mathbf{5 i}$ and $\mathbf{5 j}$ ) showed higher activity than compounds containing chloro $(\mathbf{5 c}, \mathbf{5 d})$, or bromo group $(\mathbf{5 e}, \mathbf{5 f})$. Chloro and bromo derivatives also had higher activity than other compounds. On the basis of SAR, the activity of compounds was found to depend on electron 
withdrawing nature of the substituted groups. The investigation of antimicrobial (antibacterial, antifungal and antitubercular) data revealed that the compounds $\mathbf{5 c}$, $\mathbf{5 d}, \mathbf{5 e}, \mathbf{5 f}, \mathbf{5 h}, \mathbf{5 i}$ and $\mathbf{5 j}$ displayed high activity in the series, the compounds $\mathbf{5 b}$ and 5g showed the moderate activity and the rest compounds showed less activity against all the strains compared with standard drugs. As concerning the antiinflammatory activity, the compounds $\mathbf{5 c}, \mathbf{5 d}, \mathbf{5 e}, \mathbf{5 f}, \mathbf{5 h}, \mathbf{5 i}$ and $\mathbf{5 j}$ showed the high activity while other compounds displayed moderate to lower activity.

\section{Biological study}

\section{Antibacterial, antifungal and antitubercular activities}

Series of newly synthesized compounds exhibited activity against selected microorganisms. The minimal inhibition concentrations were determined using the filter paper disc diffusion method and the concentrations had been used in $\mu \mathrm{g} / \mathrm{ml}$. All the finally synthesized compounds $\mathbf{5}(\mathbf{a}-\mathbf{j})$ had been screened in vitro for their antibacterial activity against B. subtilis, E. coli and $S$. aureus and antifungal activity against $A$. niger, $A$. flavus, $C$. albicans. The MIC values of standard Streptomycin and Griseofulvin for all bacteria and fungi were in the range of $1.25-6.25$ and $12.5-25 \mu \mathrm{g} / \mathrm{ml}$, respectively. The antitubercular activity was screened against the M. tuberculosis. For the antitubercular activity Isoniazid and Rifampicin (MIC 1.25 and $4 \mu \mathrm{g} / \mathrm{ml}$ ) were used as standards. Standards also were screened under the similar conditions for comparison (Table 1).

Table 1. Antibacterial, antifungal and antitubercular activities of compounds $5(\mathbf{a}-\mathbf{j})$

\begin{tabular}{c|c|c|c|c|c|c|c}
\hline \multirow{2}{*}{$\begin{array}{c}\text { Com- } \\
\text { pound }\end{array}$} & \multicolumn{3}{|c|}{ Antibacterial activity } & \multicolumn{3}{c|}{ Antifungal activity } & $\begin{array}{c}\text { Antitubercular } \\
\text { activity }\end{array}$ \\
\cline { 2 - 8 } & $\begin{array}{c}B . \\
\text { subtilis }\end{array}$ & $\begin{array}{c}E . \\
\text { coli }\end{array}$ & $\begin{array}{c}S . \\
\text { aureus }\end{array}$ & $\begin{array}{c}\text { A. } \\
\text { niger }\end{array}$ & $\begin{array}{c}\text { A. } \\
\text { flavus }\end{array}$ & $\begin{array}{c}\text { C. } \\
\text { albicans }\end{array}$ & $\begin{array}{c}\text { M. } \\
\text { tuberculosis }\end{array}$ \\
\hline $\mathbf{5 a}$ & 10.25 & 13.5 & 7.25 & 25 & 22.50 & 20.25 & 12.50 \\
$\mathbf{5 b}$ & 15.5 & 7.25 & 7.25 & 13.5 & 12.50 & 10.50 & 2.25 \\
$\mathbf{5 c}$ & 5.25 & 7.25 & 4.25 & 10.25 & 9.25 & 13.50 & 1.75 \\
$\mathbf{5 d}$ & 8.25 & 13.5 & 7.25 & 9.25 & 10.50 & 10.25 & 3.25 \\
$\mathbf{5 e}$ & 8.25 & 4.25 & 7.25 & 13.5 & 12.25 & 13.50 & 3.50 \\
$\mathbf{5 f}$ & 4.25 & 7.25 & 6.25 & 13.5 & 12.50 & 10.25 & 2.25 \\
$\mathbf{5 g}$ & 7.25 & 13.5 & 7.25 & 13.5 & 15.25 & 13.50 & 3.75 \\
$\mathbf{5 h}$ & 4.25 & 4.25 & 7.25 & 8.25 & 10.50 & 9.50 & 1.25 \\
$\mathbf{5 i}$ & 4.25 & 4.25 & 4.25 & 10.50 & 10.25 & 9.75 & 1.50 \\
$\mathbf{5 j}$ & 4.25 & 6.25 & 4.25 & 8.50 & 8.75 & 9.50 & 1.75
\end{tabular}

\section{Antiinflammatory activities}

Carageenan-induced rat paw oedema method was employed for evaluating the antiinflammatory activity of compounds at a dose of $50 \mathrm{mg} / \mathrm{kg}(\mathrm{b} / \mathrm{w})$ in albino rats (weighing $80-110 \mathrm{~g}$, each group contained 5 animals) using phenylbutazone as a standard drug for comparison at a dose of $30 \mathrm{mg} / \mathrm{kg}(\mathrm{b} / \mathrm{w})$. The rate paw oedema was produced by the method of Winter et al. The percentage inhibition of inflammation was calculated by applying Newbould formula. In vivo studies had been approved by institutional ethical committee, Dr. H.S. Gour University, Sagar. Results for compounds $\mathbf{5}(\mathbf{a}-\mathbf{j})$ were given in Table 2. 
Table 2. Antiinflammatory activity of compounds $5(\mathbf{a}-\mathbf{j})$

\begin{tabular}{c|c|c|c}
\hline Compound code & $\begin{array}{c}\text { Before carageenan } \\
\text { administration } \\
(\text { mean } \pm \text { SEM) }\end{array}$ & $\begin{array}{c}\text { Total increase in paw } \\
\text { volume after 5 hours } \\
\text { (mean } \pm \text { SEM) }\end{array}$ & $\begin{array}{c}\text { Percent } \\
\text { inhibition }\end{array}$ \\
\hline $\mathbf{5 a}$ & $0.60 \pm 0.02$ & $0.16 \pm 0.02$ & 50.00 \\
$\mathbf{5 b}$ & $0.64 \pm 0.02$ & $0.14 \pm 0.02$ & 56.25 \\
$\mathbf{5 c}$ & $0.66 \pm 0.02$ & $0.13 \pm 0.01$ & 59.38 \\
$\mathbf{5 d}$ & $0.68 \pm 0.02$ & $0.13 \pm 0.02$ & 59.38 \\
$\mathbf{5 e}$ & $0.66 \pm 0.03$ & $0.14 \pm 0.02$ & 56.25 \\
$\mathbf{5 f}$ & $0.65 \pm 0.02$ & $0.12 \pm 0.01$ & 62.50 \\
$\mathbf{5 g}$ & $0.67 \pm 0.02$ & $0.13 \pm 0.01$ & 59.38 \\
$\mathbf{5 h}$ & $0.64 \pm 0.03$ & $0.12 \pm 0.01$ & 62.50 \\
$\mathbf{5 i}$ & $0.65 \pm 0.02$ & $0.10 \pm 0.03$ & 68.75 \\
$\mathbf{5 j}$ & $0.67 \pm 0.03$ & $0.11 \pm 0.02$ & 65.63 \\
Control & $0.66 \pm 0.02$ & $0.32 \pm 0.01$ & - \\
Standard & $0.68 \pm 0.03$ & $0.08 \pm 0.02$ & 75.00 \\
(phenylbutazone) & & &
\end{tabular}

\section{CONCLUSIONS}

All the compounds have been synthesized giving better yields and reaction time. Antimicrobial, antitubercular and antiinflammatory activity data of compounds (shown in Table 1 and 2) have revealed that the compounds showed moderate to good activities against all the strains compared with standard drugs.

\section{Acknowledgement}

The authors are thankful to SAIF, Central Drugs Research Institute, Lucknow (India) for providing spectral and analytical data of the compounds. We are also thankful to Head of Department of Chemistry, Dr. H.S. Gour, University, Sagar (India) for giving the facilities to carryout the work.

\section{R E F ER EN C E S}

1. Upadhyay, A., Srivastava, S. K., Srivastava, S.D. (2010). Conventional and microwave assisted synthesis of some new N-[(4-oxo-2-substituted aryl-1,3-thiazolidine)acetamide]-5nitroindazoles. Eur. J. Med. Chem., 45, 3541-3548.

2. Sayyed, M., Mokle, S., Bokhare, M., Mankar, A., Surwase, S., Bhusare, S., Vibhute, Y. (2006). Synthesis of some new 2,3-diaryl-1,3-thiazolidin-4-ones as antibacterial agents. Arkivoc, 2, 187-192.

3. Mulwad, V.V., Mir, A.A. (2008). Synthesis and antibacterial screening of N-[coumarin-6yl]spiroindoloazetidin-2-ones/thiazolidin-4-ones. J. Korean Chem. Soc., 52 (6), 649-656.

4. Dua, R., Srivastava, S.K., Srivastava, S.D. (2009). Synthesis, characterization and antimicrobial activity of 4-oxothiazolidines and 5-arylidene derivatives of 2-methylimidazoles. Macroheterocycles, 2 (5), 271-274.

5. Yadav, R., Srivastava, S.D., Srivastava, S.K. (2005). Synthesis, antimicrobial and antiinflammatory activities of 4-oxothiazolidines and their 5-arylidenes. Indian J. Chem., 44B, $1262-1266$.

6. Terzioglu, N., Karalı, N., Gursoy, A., Pannecouque, C., Leysen, P., Paeshuyse, J., Neyts, J., Clercq, E. De. (2006). Synthesis and primary antiviral activity evaluation of 3-hydrazono-5nitro-2-indolinone derivatives. Arkivoc, 1, 109-118.

7. Visagaperumal, D., Kumar, R.J., Vijayaraj, R., Anbalagan, N. (2009). Microwave-induced synthesis of some new 3-substituted-1, 3-thiazolidin-4-ones for their potent antimicrobial and antitubercular activities. Int. J. Chem. Tech. Res., 4, 1048-1051. 
8. Lu, Y., Wang, Z., Li, C.-M., Chen, J., Dalton, J.T., Li, W., Miller, D.D. (2010). Synthesis, in vitro structure-activity relationship, and in vivo studies of 2-arylthiazolidine-4-carboxylic acid amides as anticancer agents. Bioorg. Med. Chem.,18 (2), 477-495.

9. Holmes, D.S., Bethell, R.C., Hann, M.M., Kitchin, J., Starkey, I.D., Storer, R. (1993). The design and synthesis of novel hydroxyproline inhibitors of HIV-1 proteinase. Bioorg. Med. Chem. Lett., 3 (8), 1485-1491.

10. Dixit, P. P., Nair, P. S., Patil, V. J., Jain, S., Arora, S. K., Sinha, N. (2005). Synthesis and antibacterial activity of novel (un)substituted benzotriazolyl oxazolidinone derivatives. Bioorg. Med. Chem. Lett., 15, 3002-3005.

11. Vora, J.J., Vasava, S.B., Patel, A.D., Parmar, K.C., Chauhan, S.K., Sharma, S.S. (2009). Synthesis, Characterization and Antibacterial Activity of a New Series of $s$-Triazines Derived with Quinolines. Eur. J. Chem., 6 (1), 201-206.

12. Barbaric, M., Kralj, M., Marjanovic, M., Husnjak, I., Pavelic, K., Filipovic-Grcic, J., Zorc, D., Zorc, B. (2007). Synthesis and in vitro antitumor effect of diclofenac and fenoprofen thiolated and nonthiolated polyaspartamide-drug conjugates. Eur. J. Med. Chem., 42, 20-29.

13. Rezaei, Z., Khabnadideh, S., Pakshir, K., Hossaini, Z., Amiri, F., Assadpour, E. (2009). Design, synthesis, and antifungal activity of triazole and benzotriazole derivatives. Eur. J. Med. Chem., 44, 3064-3067.

14. Patel, P.D., Patel, M.R., Kocsis, B., Kocsis, E., Graham, S.M., Warren, A.R., Nicholson, S.M., Billack, B., Fronczek, F.R., Talele, T.T. (2010). Design, synthesis and determination of antifungal activity of 5(6)-substituted benzotriazoles. Eur. J. Med. Chem., 45, 2214-2222.

15. Kumar, G.V.S., Rajendraprasad, Y., Mallikarjuna, B.P., Chandrashekar, S.M., Kistayya, C. (2010). Synthesis of some novel 2-substituted 5-[isopropylthiazole]-clubbed 1,2,4-triazole and 1,3,4-oxadiazoles as potential antimicrobial and antitubercular agents. Eur. J. Med. Chem., 45, 2063-2074.

16. Rajasekaran, A., Rajagopal, K.A. (2009). Synthesis of some novel triazole derivatives as antinociceptive and antiinflammatory agents. Acta Pharm., 59, 355-364.

\section{DAŽU JAUNU TIAZOLIDĪNA ATVASINĀJUMU SINTĒZE UN BIOLOĢISKĀ NOZĪME}

R. Sharma, P. Samadhiya, S.D. Srivastava, S.K. Srivastava

\section{K O P S A VIL K U M S}

Izstrādāts efektīvs cel̦š jaunas rindas N-[3-(1H-1,2,3-benzotiazol-1-il)propil2-(aizvietoto fenil)-4-okso-5-(aizvietoto benzilidēn)-1,3-tiazolidīnu karboksamīdu 5(a-j) sintēzei. Savienojumi 5(a-j) sintezēti un raksturoti ar IS, ${ }^{1} \mathrm{H}$ KMR, ${ }^{13} \mathrm{C}$ KMR, FAB masspektroskopiju un ķīmiskās analīzes datiem. Visiem galaproduktiem pārbaudīta antimikrobiālā aktivitāte pret dažām izvēlētām baktērijām, sēnītēm, kā arī prettuberkulozes (pret M. tuberculosis) un pretiekaisuma aktivitāte uz albino žurkām. 